\title{
A Computational Efficient Method for HBT Intermodulation Distortions and Two-Tone Characteristics Simulation
}

\author{
Kuen-Yu Huang, Yiming Li, C. P. Lee, and S. M. Sze \\ Department of Electronics Engineering, National Chiao Tung University \\ Hsinchu 300, Taiwan \\ ymli.ee87g@nctu.edu.tw
}

\begin{abstract}
In this paper, a new simulation method for two-tone characteristics calculations and the third-order intercept point (OIP3) of heterojunction bipolar transistor (HBT) in large-scale time domain is proposed. Base on waveform relaxation (WR) and monotone iterative (MI) methods, we solve a set of nonlinear ordinary differential equation (ODE) of equivalent circuit. With this approach, the two-tone characteristics in frequency domain for HBTs were directly computed from time domain result with fast Fourier transform (FFT). Simulation results on a realistic HBT are presented to show the accuracy and efficiency of the method.
\end{abstract}

\section{Introduction}

High power HBTs operated at high frequencies for power amplifiers have been of great interests for wireless applications in recent years [1-3]. One of the most favorite properties of the HBTs is their unusually high linearity [4-5]. The common approach to calculate the intermodulation distortion and two-tone characteristics for an HBT is to solve a set of equivalent circuit differential equations simulation in frequency domain. The harmonic balanced method is a standard approach for such problem. In circuit simulation, conventional approach to solve a set of nonlinear ODEs is the Newton's iterative method. Newton's iterative method is a local method that, in general, converges quadratically in a sufficiently small neighborhood of the exact solution.

In this paper, a novel approach for the calculation of two-tone characteristics and the OIP3 in large-scale time domain is proposed. Based on WR and MI methods, the HBT circuit equations are directly solved in time domain. The computed AC results are then analyzed with FFT to obtain the necessary information. Our new approach was using the MI method instead of the Newton's iterative method to solve the equations in time domain directly. The MI method has been successfully developed and applied to semiconductor device simulations by us earlier [6-8]. In comparison with Newton's iterative method, the major improvements of the MI method for circuit simulation are as follows: it converges globally for HBT, BJT, and MOSFET equivalent circuit models and it is readily for parallel computation. In Sec. 2, we present the model and method of calculation. Followed by results and conclusions. 


\section{A HBT Circuit Model and Method of Calculation}

As shown in Fig. 1, the simulation model is firstly formulated with nodal equations, where the Gummel-Poon large signal Model is used in the simulation [9-10]. For HBT, where the Early effect can be ignored because high doping concentration in the Base of HBT. The circuit for DC simulation and two-tone characteristic are shown in Fig. 1. First of all, the DC circuit is simulated to find the DC solutions. The result shows the accuracy of the proposed method. The similar method is then applied to simulate the circuit with a two-tone input signal. As shown in Fig.1b, $V_{\mathbb{I N}}$ is the $\mathrm{DC}$ bias and the express of $\mathrm{V}_{\text {in }}$ is as follows:

$$
V_{\text {in }}=V 1 \times \sin (2 \pi \times \text { Freq } 1 \times t)+V 2 \times \sin (2 \pi \times \text { Freq } 2 \times t),
$$

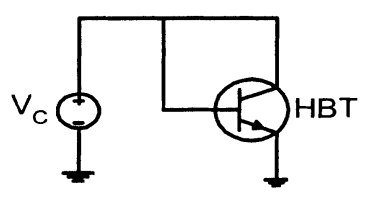

(a)

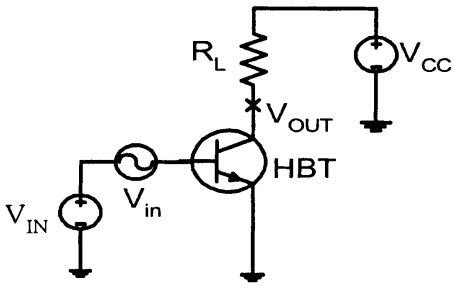

(b)

Fig. 1. (a) The circuit for DC simulation. (b) The circuit for two-tone simulation.

where the frequencies Freq 1 and Freq2 are 1.05 and $0.95 \mathrm{GHz}$, respectively. For the non-linearity of equivalent circuit, we calculate the two-tone output signal in time domain with inter-modulation. More than twenty cycles are computed with the simulator and used in FFT to obtain frequency domain analysis.

In this work, As shown in Fig. 1b, there are six ODEs have to be solved. To solve these nonlinear ODEs, our approach is: (i) Use WR method to decoupled the equations. (ii) Each decoupled ODE is then calculated with MI method. The convergence criterion for outer and MI loops are $10^{-12}$ and $10^{-8}$ in maximum error, respectively.

\section{Results and Discussions}

As shown in Fig. 2, a Gummel plot of the simulated circuit as shown in Fig. 1a is presented to show the accuracy of the proposed method. The solid lines are our results with the new simulation approach and the dotted lines are results from HSPICE. Fig. 3 demonstrates the time domain simulation of the output voltage $\left(\mathrm{V}_{\text {OUT }}\right.$, as shown in Fig. $1 \mathrm{~b}$ ) with a two-tone input signal. The simulation was done directly in time domain scale that relies on the robustness of the developed circuit simulator in this work.

As shown in Fig. 4, the spectrum of $\mathrm{V}_{\mathrm{OUT}}$ with an input voltage, $\mathrm{V}_{\text {in }}$, is plotted, where the amplitude of the input is $0.01 \mathrm{~V}$. This result was calculated from FFT with computed time domain results. Output Power of the fundamental frequency and the third order inter-modulation (IM3) as a function of input power were calculated. 
Furthermore, As shown in Fig. 5, the cross point of those two extrapolated lines is the third-order intercept point (OIP3). Higher OIP3 value presents better linearity of the device. Finally, Fig. 6 shows the OIP3 of the HBT device as a function of the collector current. The linearity of the same device varies with different biases.

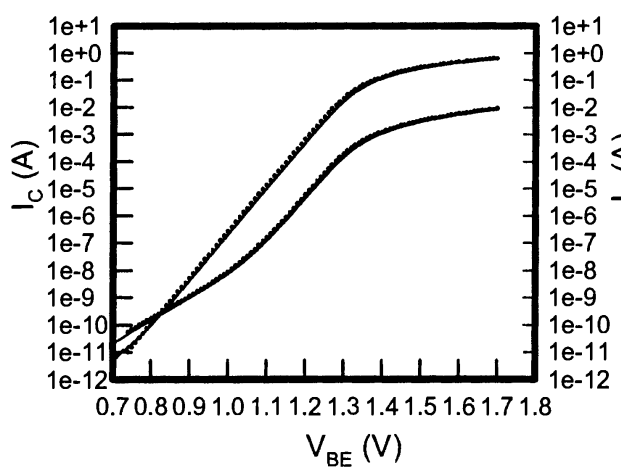

Fig. 2. Gummel plot of the HBT device.

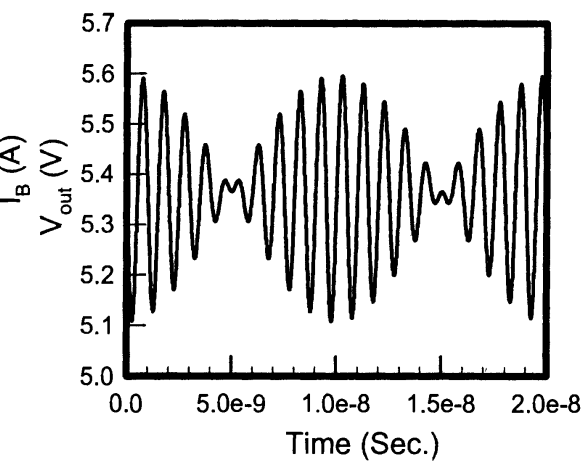

Fig. 3. Time domain simulation result of $\mathrm{V}_{\text {OUT }}$.

\section{Conclusions}

A novel circuit simulation that bases on the WR and MI methods is proposed. With this approach, the intermodulation distortion and two-tone characteristics for a HBT device is directly computed from time domain results with FFT. Simulation results on a realistic HBT are presented to show the accuracy and efficiency of the method. This method provides a new alternative in large-scaled circuit simulation. Based on the constructive monotone iterative method, the developed circuit simulator is not only convergent globally but also inherent parallel.

\section{References}

[1] Guofu N., et al. Intermodulation characteristics of UHV/CVD SiGe HBTs. Proc. Bipolar/BiCMOS Circuits and Technology Meeting, 1999; 50-53

[2] Guofu N., et al. SiGe profile design tradeoffs for RF circuit applications. IEDM Tech. Dig., 1999; 573-576

[3] Sheu S., et al. A new approach for SPICE simulation of AlGaAs/GaAs HBT subjected to burn-in test. IEEE Trans. Electron Devices, 1998; 45: 326-329

[4] Sheu S., et al. Current gain long-term instability of $\mathrm{AlGaAs} / \mathrm{GaAs}$ HBT: physical mechanism and SPICE simulation. Proc. IRPS, 1997; 248-252

[5] Chang M. F., et al. High efficiency and high linearity microwave power amplifiers based on ultra-high f/sub max/ selective buried sub-collector (SBSC) HBTs. Proc. IEEE 
MILCOM, 1998; 704-707

[6] Li Y., et al. A novel approach for the two-dimensional simulation of submicron MOSFETs using monotone iterative methodProc. IEEE VLSI-TSA, 1999;27-30

[7] Mastorakis N. Recent Advances in Applied and Theoretical Mathematics, World Scientific, 2000

[8] Li Y., et al. A New Parallel Adaptive Finite Volume Method for the Numberical Simulation of Semiconductor Devices. Ad. Prog. Tech. Dig. CCP 2000; 138

[9] Getreu L E. Modeling the Bipolar Transistor, Elsevier, New York, 1984

[10] Liu W. Handbook of III-V Heterojunction Bipolar Transistors, John Wiley \& Sons, 1998
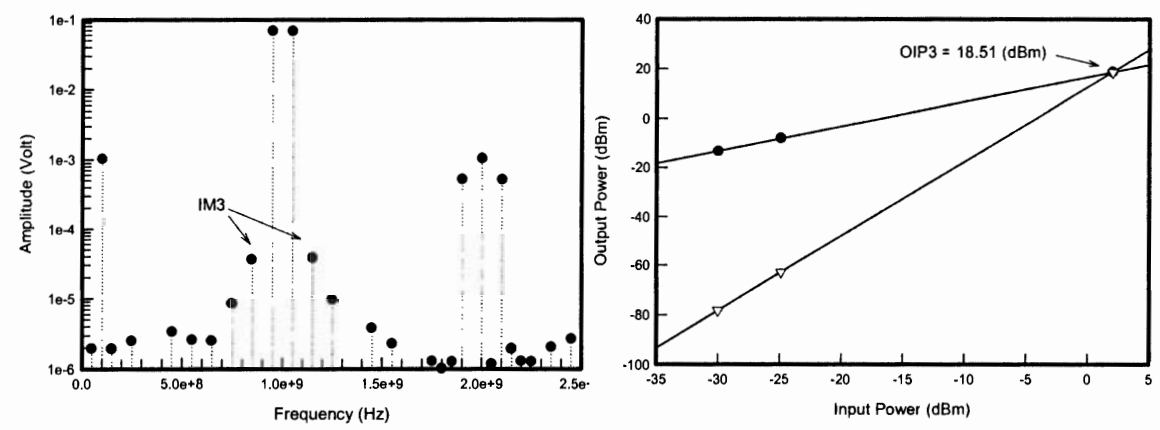

Fig. 4. The spectrum of $\mathrm{V}_{\text {OUT }}$.

Fig. 5. Plot of output Power vs. input power.

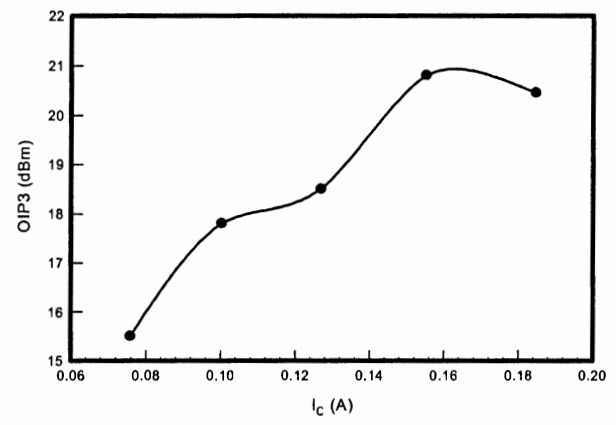

Fig. 6. OIP3 of the HBT device as a function of the $I_{C}$. 